OPEN ACCESS

Edited by:

Agustin Ibanez,

Institute of Cognitive and Translational

Neuroscience, Argentina

Reviewed by:

Adolfo M. García,

Laboratory of Experimental

Psychology and Neuroscience

(CONICET), Argentina

Rosemary Varley,

University College London, UK

${ }^{*}$ Correspondence:

Marcelo L. Berthier

mbt@uma.es

${ }^{\dagger}$ These authors have contributed equally to this work.

Received: 30 December 2016 Accepted: 17 March 2017

Published: 03 April 2017

Citation:

Berthier ML, Torres-Prioris MJ and López-Barroso D (2017) Thinking on

Treating Echolalia in Aphasia:

Recommendations and Caveats for

Future Research Directions.

Front. Hum. Neurosci. 11:164.

doi: 10.3389/fnhum.2017.00164

\section{Thinking on Treating Echolalia in Aphasia: Recommendations and Caveats for Future Research Directions}

\author{
Marcelo L. Berthier ${ }^{1 *}$, María J. Torres-Prioris ${ }^{1,2 \dagger}$ and Diana López-Barroso ${ }^{1,2 \dagger}$ \\ ${ }^{1}$ Cognitive Neurology and Aphasia Unit and Cathedra ARPA of Aphasia, Centro de Investigaciones Médico-Sanitarias and \\ Instituto de Investigación Biomédica de Málaga, University of Malaga, Malaga, Spain, ${ }^{2}$ Area of Psychobiology, Faculty of \\ Psychology, University of Malaga, Malaga, Spain
}

Keywords: imitation, repetition, echolalia, aphasia therapy, memantine

\section{ARE VERBAL IMITATION AND REPETITION THE SAME?}

Imitation in the form of repeating speech sounds, accents, and words plays a foundational role in the normal acquisition and development of language (Meltzoff et al., 2009; Adank et al., 2013) eventually contributing to a life-long fine-tuning of communication skills (Tannen, 1987; Delvaux and Soquet, 2007). Imitation of prosodic and paralinguistic features may be intentional in certain contexts (e.g., mockery, impersonation, acting rehearsal). However, in general, imitation in healthy subjects is unintended as it involves automatic mimicry of non-essential components of the acoustic-phonetic information (speaking rate, prosody, accent) embedded in the heard message (Kappes et al., 2010) - the so-called chameleon effect. Therefore, it seems that verbal imitation is not the same as verbal repetition because in the latter, the auditory stimulus is intentionally repeated and the reproduced speech contains relevant phonological information, but the incidental acoustic features of the perceived stimulus are not invariably mimicked (Kappes et al., 2009, 2010).

\section{ECHOLALIC REPETITION AND ITS SUBTYPES}

Echolalia, the repetition of words and/or utterances spoken by another person (Wallesch, 1990), is frequently documented in individuals with autism spectrum disorders (Stiegler, 2015), neurodegenerative dementias (Da Cruz, 2010; Kertesz et al., 2010), post-stroke aphasia (Geschwind et al., 1968; Christman et al., 2004), and other neurologic and psychiatric disorders (Berthier et al., 2017a). However, there are no studies on the prevalence of echolalia in these conditions. This is intriguing as, for instance, echolalia is a usual accompanying feature of transcortical aphasias, which represent $4-20 \%$ of all aphasias (Berthier, 1999). Moreover, echolalia has occasionally been described during the recovery process of classical perisylvian aphasias (global, Wernicke, conduction, Broca; Brown, 1975; Hadano et al., 1998; López-Barroso et al., 2017). This implies that a more in depth assessment would inflate the prevalence rates.

Echolalia is a heterogeneous symptom of aphasia and several subtypes have been described (Wallesch, 1990; Berthier, 1999). More than one type of echolalia can coexist in the same patient (Brown, 1975; Hadano et al., 1998) and changes from one form to another (i.e., from complete to partial) during aphasia evolution is common. The most severe types of echolalia occur in aphasias with preserved repetition abilities (transcortical aphasias; Berthier et al., 2017a). Two of them, 
ambient echolalia ${ }^{1}$ and echoing approval ${ }^{2}$ are chiefly characterized by the production of echoes of comments and questions not directed to the patient but to other people. Such disinhibition, elicited by merely hearing speech in the environment, results from diffuse brain injury (Geschwind et al., 1968) or extensive unilateral or bilateral lesions in medial frontal and anterior cingulate cortices and subcortical structures (Ghika et al., 1996; Suzuki et al., 2009, 2012). In both forms, deficient inhibition of repetition conceivably results from altered control of shared representations (misunderstanding the intentions of others; Frith and Frith, 2006; Brass et al., 2009; Besnard et al., 2011) and evaluation of outcomes (e.g., impaired reflection on one's own performance; Passingham et al., 2010; Berthier et al., 2017a). At variance with the abovementioned types of verbal echoing, another severe form named automatic echolalia ${ }^{3}$ is provoked when patients are directly addressed, and not when comments and questions are directed to other people. This suggests better control of shared representations (self-other distinctions). Awareness about the irrepressible echoing may or may not be preserved, but note that these cognitive domains have not been formally investigated so far. Automatic echolalia in aphasia usually occurs after lesions in the left hemisphere placed outside the perisylvian language area (PLA; the isolation of the speech area hypothesis) responsible for verbal repetition. This hypothesis, early championed by Goldstein (1948) and Geschwind (Geschwind et al., 1968), maintains that echolalic repetition in aphasia occurs because the left PLA is anatomically intact, but out-of-control by virtue of being disconnected from close and distant eloquent cortical regions (SMA, temporo-parietal cortex) underlying language production and comprehension. Nevertheless, other mechanisms (right hemisphere or bilateral hypotheses) underlying echolalic repetition have been proposed (Niessl von Mayendorf, 1911; Brown, 1975). Modern studies provided evidence that in such cases the left PLA area may be dysfunctional (Berthier et al., 1997) with limited competence to generate verbal repetition and echolalia (López-Barroso et al., 2017). In this situation, verbal echoing most likely depends on the vicarious activity of the right hemisphere (López-Barroso et al., 2017). In support, studies in healthy volunteers using functional neuroimaging (Saur et al., 2008) and transient virtual lesions over the left inferior frontal gyrus (Hartwigsen et al., 2013) revealed bilateral temporofrontal participation during repetition of words and increased activity in the contralateral homologous area during repetition of nonwords, respectively. Another piece of evidence that supports the right hemisphere hypothesis is the case of formerly globally aphasic patients with large left PLA lesions, who develop automatic echolalia years after aphasia onset

\footnotetext{
${ }^{1}$ The term ambient echolalia (Fisher, 1988) is applied when patients repeat words and sentences coming from unrelated conversations around them even when people are talking in a nearby room (Suzuki et al., 2012).

${ }^{2}$ The term echoing approval is used for those patients who imitate the affirmative or negative syntactical construction of questions or the intonation patterns even when questions are directed to other persons (environmental-dependency syndrome; Ghika et al., 1996).

${ }^{3}$ Automatic echolalia refers to the production of echoes in an impulsive, "parrotlike" manner. Patients do not appear inhibited by any type of verbal information including non-words or foreign languages.
}

through gradual remodeling of right hemisphere networks (Pulvermüller and Schönle, 1993; Berthier et al., 1997). In keeping with these findings, a right intracarotid amobarbital injection (Wada test) suppressed automatic echolalia in a case of transcortical sensory aphasia and left hemisphere damage (Case 1 in Berthier et al., 1991). In this regard, what requires elucidation is why only a small proportion of patients with severe aphasia after left extensive hemisphere damage develop echolalia. A tentative explanation is that individual differences in the status of repetition, and hence on the possibility of developing verbal echoing in aphasia, may depend on both the premorbid structure of gray matter (Xing et al., 2016) and variability of right white matter tracts (Catani et al., 2007; Berthier et al., 2012; Forkel et al., 2014).

Two less severe forms of verbal echoing have been designated as mitigated echolalia ${ }^{4}$ (Pick, 1924; Lebrun et al., 1971) and effortful echolalia ${ }^{5}$ (Hadano et al., 1998). Information on these variants is scarce, but one distinctive element is that they are also observed in aphasias with impaired verbal repetition (conduction aphasia, Wernicke's aphasia, Broca's aphasia). The responsible lesions involve the left temporo-parietal cortex in mitigated echolalia and large portions of the left PLA in effortful echolalia. It is apparent that mitigated echolalia entails better control over the echoed material than in the more severe forms as reflected by the introduction of changes in the reproduced emissions compared to the verbatim repetition that accompanies, for example, automatic echolalia. Modifications in wording or intonation on the echoed emissions may have different purposes such as recapitulate meaning, regain attention, take time to plan a response, reinforcement of an idea, contradict, complement the just received message, or empathize with the interlocutor. However, despite the general consensus that the production of echoes of words and phrase fragments is aimed to resolve impaired access to word meaning during auditory comprehension, deficits in auditory-verbal short-term memory and incompetent inhibitory control have also been described (Berthier et al., 2017b). Thus, it seems that mitigated echolalia is not always in the service of improving auditory comprehension.

Little information also exists on the other type, effortful echolalia. It is essentially a form of mitigated echolalia, yet the production of echoes is laborious and limited to short phrase fragments produced with dysarthria and distorted prosody (Hadano et al., 1998). At present, there is no information on whether effortful echolalia helps the very limited communication ability or whether it merely represents a disinhibition symptom. In the few cases reported up to now, effortful echolalia results from simultaneous involvement of the left supplementary motor area and left PLA (e.g., Broca's area, anterior insula; Hadano et al., 1998). While verbal echoes after damage to the left supplementary motor area in other forms of echolalia are produced with fluent and well-articulated speech, the laborious production in effortful echolalia reflects the additional

\footnotetext{
${ }^{4}$ Mitigated echolalia refers to any language change in the echoed emission for communicative purposes (Pick, 1924), but recent data suggest that this is not always the case (Berthier et al., 2017b).

${ }^{5}$ Effortful echolalia denotes the articulatory struggling, distorted prosody, and increased effort observed in the echoes (Hadano et al., 1998).
} 
involvement of the left anterior PLA. Awareness on the irrepressible character of echoes seems to be variable and needs further evaluation.

\section{NEURAL MECHANISMS}

Nowadays the neural mechanisms supporting verbal imitation/repetition (Mashal et al., 2012) and their inhibition in inappropriate situations (Bien et al., 2009; Aron et al., 2014) are relatively well-known. Progress in the study of network models for action observation and imitation of speech in healthy subjects suggest that action understanding, imitation, and verbal learning requires an orchestrated coordination of different brain region in which the mirror neuron system (MNS) and the white matter tracts linking its different nodes are involved (Kohler et al., 2002; Arbib, 2010; but see criticisms to the role of MNS in Hickok, 2009; Mikulan et al., 2014). The audio-visual MNS is located in ventrolateral prefrontal cortex, superior temporal gyrus, and inferior parietal lobule overlapping with the dorsal speech-processing stream, and these areas are linked via the arcuate fasciculus (Arbib, 2010; Corballis, 2010). The audiovisual MNS represents a mechanism for integrating perception and action, which fits well with preferential role of the dorsal stream, involved in automatic non-semantic translation from the sensory to the motor code (i.e., auditory-motor integration), required for voluntary verbal repetition, short-term memory, and verbal learning (Hickok and Poeppel, 2007; RodríguezFornells et al., 2009; López-Barroso et al., 2013, 2015). This intricate neural system operates under the supervision of a bilateral executive-control network (premotor, posterior parietal and frontal-parietal opercular cortices, right inferior frontal, and superior temporal cortices, and basal ganglia), which acts as a "brake" supressing inappropriate, automatic overt repetition (echolalia; Aron et al., 2014; Bien et al., 2009). When brain pathology abolishes the regulatory function of these areas in the left hemisphere of patients with aphasia, their verbal repetition is out of control and echolalia ensues by virtue of automatic activation of action-perception circuits including the audio-visual MNS (Berthier et al., 2006, 2017a). The mechanism is possibly more complex in bilingual and polyglot patients with aphasia who repeat a just heard verbal material but in a different language [examiner: "What time is it?"; patient response: "Quelle heure est-il?” (Veyrac, 1931; see review in García, 2015)]. Nevertheless, imitation of paralinguistic features (prosody) is not always possible and repeated words and sentences sound flat and devoid of emotional coloring (Speedie et al., 1984; Berthier et al., 1996; Kappes et al., 2009), thus suggesting that repetition and imitation are dissociable. Alternatively, less severe forms of echolalia are produced in a voluntary manner and thus are not directly associated to a grossly abnormal functioning of this regulatory system.

\section{BROADENING THE SCOPE OF TESTING FOR ECHOLALIA}

The notion that impairments in non-language cognitive domains and behavior influence the clinical presentation and evolution of aphasia is gaining credence amongst aphasiologists (Kauhanen et al., 2000; Fucetola et al., 2006; van de Sandt-Koenderman et al., 2008; Lambon Ralph et al., 2010; El Hachioui et al., 2014). Since the same argument probably holds for echolalia in aphasia, we emphasize the strong necessity to explore the relationship between verbal echoing, concurrent deficits in language and high-level cognitive non-language processes, and the neural mechanisms underpinning these domains in aphasia. Our proposal is that the analysis of this interaction would provide hints for devising neurorehabilitation strategies tailored to each patient needs, trying to be consistent with the current function of echolalia and its potential instrumental role in relation to functional communication.

Through the years, it has been advocated that deficits underpinning echolalia are related to breakdown of various domains including inhibitory control, mentalizing (theory of mind), decision making, awareness, auditory comprehension, auditory-visual feedback, and auditory-verbal short-term memory (see Berthier et al., 2017a). Nevertheless, the relative contribution of each deficit to the different types of echolalia remains unexplored. The Figure 1 summarizes the existing types of echolalia together with a proposal of the non-linguistic cognitive and behavioral functions that could be involved in this complex symptom and that we suggest to explore in each type.

\section{DO ALL TYPES OF ECHOLALIA REQUIRE TREATMENT?}

One key issue that needs elucidation is whether all types of echolalia associated with aphasia require treatment. Moreover, in the case that one advocates a therapeutic intervention for echolalia, the question is what to do with it, inhibit or reshaping? The answer of this largely unexplored issue is far for being contested and is probably more complex than it may appear. Echolalia is a symptom that appears in a wider clinical context, very often presented together with comprehension, fluency or short-term memory deficits, amongst other nonlanguage cognitive deficits (Figure 1). In addition, the degree of control over the repeated material is subjected to changes on a severity continuum from an uncontrollable automatic echoing to a more indolent voluntary repetition. This would imply that the faulty inhibition of impulsive echolalia seen in some cases contrasts sharply with the voluntariness to repeat verbal material seen in other cases, mostly to improve auditory comprehension, in which the content of verbal echoes is not always a verbatim reproduction of what has been heard. This poses the question of how and when echoes have unfavorable or beneficial effects on aphasia. The extant evidence suggests that there are not determinant answers that will be suitable for all cases. The whole clinical profile of each patient should be considered. The more severe variants of echolalia are often highly disruptive and need to be directly targeted in the rehabilitation process. Nevertheless, the evidence suggests that in cases wherein automatic echolalia in non-fluent transcortical aphasias is the only available channel for verbal production, efforts to redirect and incorporate echoes in the service of speech production and comprehension using therapies tailored to modulate the 


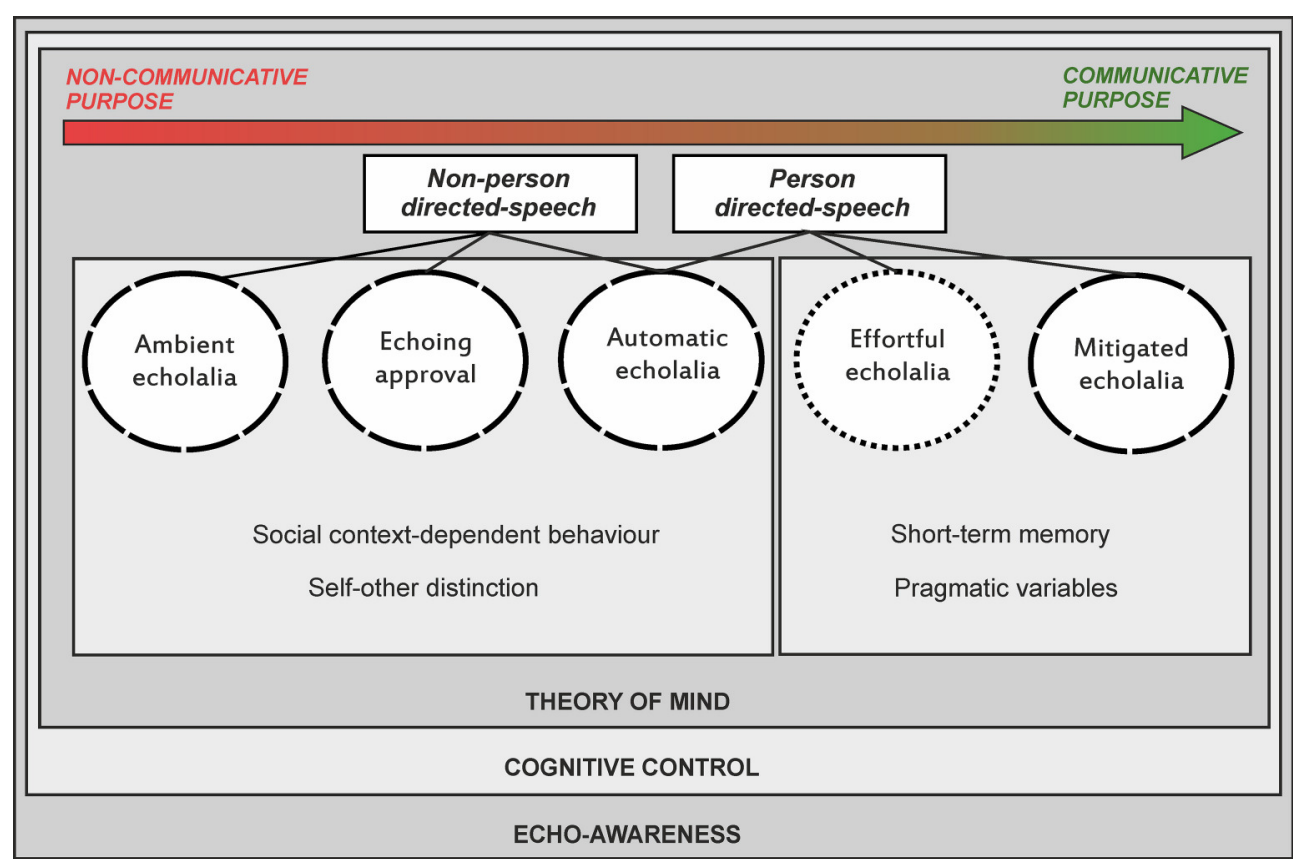

CFluent echolalia

Non-fluent echolalia

FIGURE 1 | A scheme depicting the continuum of severity of echolalia types ranging from severe (non-communicative purpose) to mild (communicative purpose) forms together with the identification of precipitants stimuli (environmental or personal). The Figure also summarizes a proposal of non-linguistic cognitive functions (e.g., self-other distinction, pragmatic variables) that could be evaluated in each type to help identify the diverse deficits underlying echolalia and the development of treatment strategies. Evaluation is also required in potential deficits affecting theory of mind, cognitive control, and echo-awareness, which are probably more generally involved in all forms. A distinction between types of echolalia produced with fluent and effortless pattern and another emitted with non-fluent and laborious speech is also shown.

activity of action-perception links (e.g., Constraint-Induced Aphasia Therapy-CIAT) are useful (Pulvermüller and Schönle, 1993; Kurland et al., 2012). The picture is not as clear for the less severe types (mitigated and effortful). Even when, in many cases, echolalia may be functional and, for example, facilitate comprehension, the incessant repetition of auditory stimuli may interfere with functional communication and make evaluations excessively long (Berthier et al., 2017b). In a recent single case study of a patient with residual Wernicke's aphasia, mitigated echolalia was significantly reduced using CIAT (supplemented with verbal instructions made by the therapist to attenuate imitative tendencies) and a cognitive-enhancing drug (memantine; Berthier et al., 2017b).

The recent identification of a neural network for action observation and imitation of speech (Mashal et al., 2012) provided a theoretical framework for developing new modelbased therapies for aphasia, namely IMITATE (Intensive Mouth Imitation and Talking for Aphasia Therapeutic Effects; Lee et al., 2010; Sarasso et al., 2014; Duncan and Small, 2016) and SPEECH ENTRAINMENT (Fridriksson et al., 2012). These interventions aim to improve speech production through action observation and audio-visual feedback via verbal repetitionimitation, which recruits the dorsal and ventral streams in both cerebral hemispheres (Lee et al., 2010; Fridriksson et al., 2012; Sarasso et al., 2014; Chen et al., 2015). Preliminary evidence indicates that this treatment approach facilitate recovery of speech production in different types of aphasia, including in cases of non-fluent transcortical aphasias, which are usually associated with echolalia, by inducing plastic changes in both cerebral hemispheres (Sarasso et al., 2014; Chen et al., 2015). However, these preliminary studies did not clarify if treated patients actually had echolalia. This is important because non-invasive overstimulation of the MNS in the left inferior frontal gyrus facilitates verbal repetition (Restle et al., 2012) and stimulation of fronto-median areas, which exerts a top-down inhibitory control over the MNS, induces echophenomena (Finis et al., 2013). Therefore, it remains to be determined whether therapies like IMITATE and SPEECH ENTRAINMENT tailored to strengthen the activity of the MNS are applicable to aphasic patients with echolalia. In any case, more studies are needed to determine whether reshaping the activity of the observation-imitation networks may redirect echolalia to the service of spontaneous speech in cases of non-fluent aphasias.

\section{CONCLUSIONS}

In this opinion article, we have analyzed the current stateof-the-art of echolalia in aphasia. We aimed to enlighten some recommendations to gain insight on diagnosis, neural mechanisms, and treatment of echolalia as well as to call 
attention on caveats that merit attention and analysis. Studies of prevalence are warranted because echolalia is very frequent in degenerative dementias coursing with aphasia (Alzheimer's disease, semantic dementia) and because neuropharmacological interventions can attenuate these symptoms in patients with Alzheimer's disease (Asp et al., 2006). Understanding the relationship of the different types of echolalia with aphasia is paramount to design adequate methodology for assessment and treatment strategies. At present, the analyzed data suggest that echolalia interfering with functional communication should be inhibited, whereas when echolalia is the only available verbal channel in aphasic cases with non-fluent speech it could be redirected to gradually convert such disinhibited speech into a meaningful communicative function.

\section{REFERENCES}

Adank, P., Stewart, A. J., Connell, L., and Wood, J. (2013). Accent imitation positively affects language attitudes. Front. Psychol. 4:280. doi: 10.3389/fpsyg.2013.00280

Arbib, M. A. (2010). Mirror system activity for action and language is embedded in the integration of dorsal and ventral pathways. Brain Lang. 112, 12-24. doi: 10.1016/j.bandl.2009.10.001

Aron, A. R., Robbins, T. W., and Poldrack, R. A. (2014). Inhibition and the right inferior frontal cortex: one decade on. Trends Cogn. Sci. 18, 177-185. doi: $10.1016 /$ j.tics.2013.12.003

Asp, E., Cloutier, F., Fay, S., Cook, C., Robertson, M. L., Fisk, J., et al. (2006). Verbal repetition in patients with Alzheimer's disease who receive donepezil. Int. J. Geriatr. Psychiatry 21, 426-431. doi: 10.1002/gps.1486

Berthier, M. L. (1999). "Chapter 6, Echophenomena, automatic speech and prosody," in Transcortical aphasias, ed M. L. Berthier (Hove: Psychology Press), 151-186.

Berthier, M. L., Dávila, G., and Torres-Prioris, M. J. (2017a). "Echophenomena in aphasia: Causal mechanisms and clues for intervention," To appear in Aphasia Rehabilitation: Clinical Challenges, eds P. Coppens and J. Patterson (Burlington, MA: Jones \& Bartlett Learning), 143-172.

Berthier, M. L., Fernández, A. M., Martínez-Celdrán, E., and Kulisevsky, J. (1996). Perceptual and acoustic correlates of affective prosody repetition in transcortical aphasias. Aphasiology 10, 711-721. doi: 10.1080/0268703960 8248445

Berthier, M. L., Lambon Ralph, M. A., Pujol, J., and Green, C. (2012). Arcuate fasciculus variability and repetition: the left sometimes can be right. Cortex 48 , 133-143. doi: 10.1016/j.cortex.2011.06.014

Berthier, M. L., Posada, A., Puentes, C., and Hinojosa, J. (1997). Brain SPECT imaging in transcortical aphasias: the functional status of the left perisylvian language cortex. Eur. J. Neurol. 4, 551-560. doi: 10.1111/j.1468-1331.1997.tb00405.x

Berthier, M. L., Pulvermüller, F., Green, C., and Higueras, C. (2006). Are release phenomena explained by disinhibited mirror neuron circuits? Arnold Pick's remarks on echographia and their relevance for modern cognitive neuroscience. Aphasiology 20, 462-480. doi: 10.1080/026870305004 84004

Berthier, M. L., Starkstein, S. E., Leiguarda, R., Ruiz, A., Mayberg, H. S., Wagner, H., et al. (1991). Transcortical aphasia. Importance of the nonspeech dominant hemisphere in language repetition. Brain 114, 1409-1427. doi: 10.1093/brain/114.3.1409

Berthier, M. L., Torres-Prioris, M. J., López-Barroso, D., Thurnhofer-Hemsi, K., Paredes-Pacheco, J., Roé-Vellvé, N., et al. (2017b). Are you a doctor? Are you a doctor? I'm not a doctor! A reappraisal of mitigated echolalia in aphasia with evaluation of neural correlates and treatment approaches. Aphasiology. doi: 10.1080/02687038.2016.1274875. [Epub ahead of print].

Besnard, J., Allain, P., Aubin, G., Chauviré, V., Etcharry-Bouyx, F., and Le Gall, D. (2011). A contribution to the study of environmental dependency

\section{AUTHOR CONTRIBUTIONS}

All authors listed, have made substantial, direct, and intellectual contribution to the work, and approved it for publication. MB, MT, and DL drafted the article and revised it critically for important intellectual content.

\section{ACKNOWLEDGMENTS}

MT has been funded by a $\mathrm{PhD}$ scholarship from the Spanish Ministry of Education, Culture and Sport under the FPU program (FPU14/04021). DL has been supported by the "Juan de la Cierva" program of the Spanish Ministry of Economy and Competitiveness (FJCI-2014-22953).

phenomena: the social hypothesis. Neuropsychologia 49, 3279-3294. doi: 10.1016/j.neuropsychologia.2011.08.001

Bien, N., Roebroeck, A., Goebel, R., and Sack, A. T. (2009). The brain's intention to imitate: the neurobiology of intentional versus automatic imitation. Cereb. Cortex 19, 2338-2351. doi: 10.1093/cercor/bhn251

Brass, M., Ruby, P., and Spengler, S. (2009). Inhibition of imitative behaviour and social cognition. Philos. Trans. R. Soc. Lond. B. Biol. Sci. 364, 2359-2367. doi: $10.1098 /$ rstb.2009.0066

Brown, J. W. (1975). The problem of repetition: a study of "conduction" aphasia and the "isolation" syndrome. Cortex 11, 37-52. doi: 10.1016/S0010-9452(75)80019-0

Catani, M., Allin, M. P., Husain, M., Pugliese, L., Mesulam, M. M., Murray, R. M., et al., (2007). Symmetries in human brain language pathways correlate with verbal recall. Proc. Natl. Acad. Sci. U.S.A. 104, 17163-17168. doi: 10.1073/pnas.0702116104

Chen, W., Ye, Q., Ji, X., Zhang, S., Yang, X., Zhou, Q., et al. (2015). Mirror neuron system based therapy for aphasia rehabilitation. Front. Psychol. 6:1665. doi: 10.3389/fpsyg.2015.01665

Christman, S. S., Boutsen, F. R., and Buckingham, H. W. (2004). Perseveration and other repetitive verbal behaviors: functional dissociations. Semin. Speech Lang. 25, 295-307. doi: 10.1055/s-2004-837243

Corballis, M. C. (2010). Mirror neurons and the evolution of language. Brain Lang. 112, 25-35. doi: 10.1016/j.bandl.2009.02.002

Da Cruz, F. M. (2010). Verbal repetitions and echolalia in Alzheimer's discourse. Clin. Linguist. Phon. 24, 848-858. doi: 10.3109/02699206.2010.511403

Delvaux, V., and Soquet, A. (2007). The influence of ambient speech on adult speech productions through unintentional imitation. Phonetica 64, 145-173. doi: $10.1159 / 000107914$

Duncan, E. S., and Small, S. L. (2016). "Chapter 84, Imitation-based aphasia therapy," in Neurobiology of Language, eds G. Hickok and S. L. Small (London: Academic Press), 1055-1065

El Hachioui, H., Visch-Brink, E. G., Lingsma, H. F., van de Sandt-Koenderman, M. W., Dippel, D. W., Koudstaal, P. J., et al. (2014). Nonlinguistic cognitive impairment in poststroke aphasia: a prospective study. Neurorehabil. Neural Repair 28, 273-281. doi: 10.1177/1545968313508467

Finis, J., Enticott, P. G., Pollok, B., Münchau, A., Schnitzler, A., and Fitzgerald, P. B. (2013). Repetitive transcranial magnetic stimulation of the supplementary motor area induces echophenomena. Cortex 49, 1978-1982. doi: 10.1016/j.cortex.2012.08.019

Fisher, C. M. (1988). Neurologic fragments. I. Clinical observations in demented patients. Neurology 38, 1868-1873. doi: 10.1212/WNL.38.12.1868

Forkel, S. J., Thiebaut de Schotten, M., Dell'Acqua, F., Kalra, L., Murphy, D. G., Williams, S. C., et al. (2014). Anatomical predictors of aphasia recovery: a tractography study of bilateral perisylvian language networks. Brain 137, 2027-2039. doi: 10.1093/brain/awu113

Fridriksson, J., Hubbard, H. I., Hudspeth, S. G., Holland, A. L., Bonilha, L., Fromm, D., et al. (2012). Speech entrainment enables patients with Broca's aphasia to produce fluent speech. Brain 135, 3815-3829. doi: 10.1093/brain/aws301 
Frith, C. D., and Frith, U. (2006). The neural basis of mentalizing. Neuron 50, 531-534.

Fucetola, R., Connor, L. T., Perry, J., Leo, P., Tucker, F. M., and Corbetta, M. (2006). Aphasia severity, semantics, and depression predict functional communication in acquired aphasia. Aphasiology 20,449-461. doi: 10.1080/02687030500390177

García, A. M. (2015). Translating with an injured brain: neurolinguistic aspects of translation as revealed by bilinguals with cerebral lesions. Meta Trans. J. 60, 112-134. doi: 10.7202/1032402ar

Geschwind, N., Quadfasel, F. A., and Segarra, J. M. (1968). Isolation of speech area. Neuropsychologia 6, 327-340. doi: 10.1016/0028-3932(68)90005-5

Ghika, J., Bogousslavsky, J., Ghika-Schmid, F., and Regli, F. (1996). "Echoing approval": a new speech disorder. J. Neurol. 243, 633-637. doi: $10.1007 /$ BF00878658

Goldstein, K. (1948). "Pictures of speech disturbances due to impairment of the non-language mental performances," in Language and Language Disturbances, (New York, NY: Grune \& Stratton), 292-309.

Hadano, K., Nakamura, H., and Hamanaka, T. (1998). Effortful echolalia. Cortex 34, 67-82. doi: 10.1016/S0010-9452(08)70737-8

Hartwigsen, G., Saur, D., Price, C. J., Ulmer, S., Baumgaertner, A., and Siebner, H. R. (2013). Perturbation of the left inferior frontal gyrus triggers adaptive plasticity in the right homologous area during speech production. Proc. Natl. Acad. Sci. U.S.A. 110, 16402-16407. doi: 10.1073/pnas.1310190110

Hickok, G. (2009). The role of mirror neurons in speech and language processing. Brain Lang. 112, 1-2. doi: 10.1016/j.bandl.2009.10.006

Hickok, G., and Poeppel, D. (2007). The cortical organization of speech processing. Nat. Rev. Neurosci. 8, 393-402. doi: 10.1038/nrn2113

Kappes, J., Baumgaertner, A., Peschke, C., and Ziegler, W. (2009). Unintended imitation in nonword repetition. Brain Lang. 111, 140-151. doi: 10.1016/j.bandl.2009.08.008

Kappes, J., Baumgaertner, A., Peschke, C., Goldenberg, G., and Ziegler, W. (2010). Imitation of para-phonological detail following left hemisphere lesions. Neuropsychologia 48, 1115-1124. doi: 10.1016/j.neuropsychologia.2009.12.012

Kauhanen, M. L., Korpelainen, J. T., Hiltunen, P., Määttä, R., Mononen, H., Brusin, E., et al. (2000). Aphasia, depression, and non-verbal cognitive impairment in ischaemic stroke. Cerebrovasc. Dis. 10, 455-461. doi: 10.1159/0000 16107

Kertesz, A., Jesso, S., Harciarek, M., Blair, M., and McMonagle, P. (2010). What is semantic dementia? a cohort study of diagnostic features and clinical boundaries. Arch. Neurol. 67, 483-489. doi: 10.1001/archneurol. 2010.55

Kohler, E., Keysers, C., Umiltà, M. A., Fogassi, L., Gallese, V., and Rizzolatti, G. (2002). Hearing sounds, understanding actions: action representation in mirror neurons. Science 297, 846-848. doi: 10.1126/science.1070311

Kurland, J., Pulvermüller, F., Silva, N., Burke, K., and Andrianopoulos, M. (2012). Constrained versus unconstrained intensive language therapy in two individuals with chronic, moderate-to-severe aphasia and apraxia of speech: behavioral and fMRI outcomes. Am. J. Speech Lang. Pathol. 21, S65-S87. doi: 10.1044/1058-0360(2012/11-0113)

Lambon Ralph, M. A., Snell, C., Fillingham, J. K., Conroy, P., and Sage, K. (2010). Predicting the outcome of anomia therapy for people with aphasia post CVA: both language and cognitive status are key predictors. Neuropsychol. Rehabil. 20, 289-305. doi: 10.1080/09602010903237875

Lebrun, Y., Rubio, S., Jongen, E., and Demol, O. (1971). On echolalia, echo-answer, and contamination. Acta Neurol. Belg. 71, 301-308.

Lee, J., Fowler, R., Rodney, D., Cherney, L., and Small, S. L. (2010). IMITATE: an intensive computer-based treatment for aphasia based on action observation and imitation. Aphasiology 24, 449-465. doi: 10.1080/02687030802714157

López-Barroso, D., Catani, M., Ripollés, P., Dell'Acqua, F., Rodríguez-Fornells, A., and de Diego-Balaguer, R. (2013). Word learning is mediated by the left arcuate fasciculus. Proc. Natl. Acad. Sci. U.S.A. 110, 13168-13173. doi: $10.1073 /$ pnas. 1301696110

López-Barroso, D., Ripollés, P., Marco-Pallarés, J., Mohammadi, B., Münte, T. F., Bachoud-Lévi, A. C., et al. (2015). Multiple brain networks underpinning word learning from fluent speech revealed by independent component analysis. Neuroimage 110, 182-193. doi: 10.1016/j.neuroimage.2014.12.085

López-Barroso, D., Torres-Prioris, M. J., Roé-Vellvé, N., Thurnhofer-Hemsi, K., J., Paredes-Pacheco, J., et al. (2017). "A reappraisal of echolalia in aphasia: A case-series study with multimodal neuroimaging," in Presented at the XXXV European Workshop of Cognitive Neuroscience. Bressanone.
Mashal, N., Solodkin, A., Dick, A. S., Chen, E. E., and Small, S. L. (2012). A network model of observation and imitation of speech. Front. Psychol. 3:84. doi: 10.3389/fpsyg.2012.00084

Meltzoff, A. N., Kuhl, P. K., Movellan, J., and Sejnowski, T. J. (2009). Foundations for a new science of learning. Science 325, 284-288. doi: $10.1126 /$ science.1175626

Mikulan, E. P., Reynaldo, L., and Ibáñez, A. (2014). Homuncular mirrors: misunderstanding causality in embodied cognition. Front. Hum. Neurosci. 8:299. doi: 10.3389/fnhum.2014.00299

Niessl von Mayendorf, E. (1911). Die aphasischen Symptome und ihre corticale Lokalisation. Leipzig: Barth.

Passingham, R. E., Bengtsson, S. L., and Lau, H. C. (2010). Medial frontal cortex: from self-generated action to reflection on one's own performance. Trends Cogn. Sci. 14, 16-21. doi: 10.1016/j.tics.2009.11.001

Pick, A. (1924). On the pathology of echographia. Brain 47, 417-429.

Pulvermüller, F., and Schönle, P. W. (1993). Behavioral and neuronal changes during treatment of mixed transcortical aphasia: a case study. Cognition 48, 139-161. doi: 10.1016/0010-0277(93)90028-T

Restle, J., Murakami, T., and Ziemann, U. (2012). Facilitation of speech repetition accuracy by theta burst stimulation of the left posterior inferior frontal gyrus. Neuropsychologia 50, 2026-2031. doi: 10.1016/j.neuropsychologia.2012.05.001

Rodríguez-Fornells, A., Cunillera, T., Mestres-Missé, A., and de Diego-Balaguer, R. (2009). Neurophysiological mechanisms involved in language learning in adults. Philos. Trans. R. Soc. Lond. B. Biol. Sci. 364, 3711-3735. doi: 10.1098/rstb.2009.0130

Sarasso, S., Määttä, S., Ferrarelli, F., Poryazova, R., Tononi, G., and Small, S. L. (2014). Plastic changes following imitation-based speech and language therapy for aphasia: a high-density sleep EEG study. Neurorehabil. Neural Repair 28, 129-138. doi: 10.1177/1545968313498651

Saur, D., Kreher, B. W., Schnell, S., Kümmerer, D., Kellmeyer, P., Vry, M. S., et al. (2008). Ventral and dorsal pathways for language. Proc. Natl. Acad. Sci. U.S.A. 105, 18035-18040. doi: 10.1073/pnas.0805234105

Speedie, L. J., Coslett, H. B., and Heilman, K. M. (1984). Repetition of affective prosody in mixed transcortical aphasia. Arch. Neurol. 41, 268-270. doi: 10.1001/archneur.1984.04050150046014

Stiegler, L. N. (2015). Examining the echolalia literature: where do speechlanguage pathologists stand? Am. J. Speech Lang. Pathol. 24, 750-762. doi: 10.1044/2015_AJSLP-14-0166

Suzuki, T., Itoh, S., Arai, N., Kouno, M., Noguchi, M., Takatsu, M., et al. (2012). Ambient echolalia in a patient with germinoma around the bilateral ventriculus lateralis: a case report. Neurocase 18, 330-335. doi: 10.1080/13554794.2011.608364

Suzuki, T., Itoh, S., Hayashi, M., Kouno, M., and Takeda, K. (2009). Hyperlexia and ambient echolalia in a case of cerebral infarction of the left anterior cingulate cortex and corpus callosum. Neurocase 15, 384-389. doi: 10.1080/13554790902842037

Tannen, D. (1987). Repetition in conversation: toward a poetics of talk. Language 63, 574-605. doi: 10.2307/415006

van de Sandt-Koenderman, W. M. E., van Harskamp, F., Duivenvoorden, H. J., Remerie, S. C., van der Voort-Klees, Y. A., Wielaert, S. M., et al. (2008). MAAS (Multi-Axial Aphasia System): realistic goal setting in aphasia rehabilitation. Int. J. Speech Lang. Pathol. 31, 314-320. doi: 10.1097/mrr.0b013e3282fc0f23

Veyrac, G.-J. (1931). Étude de l'aphasie Chez les Sujets Polyglottes. Paris: L. Arnette. Wallesch, C. W. (1990). Repetitive verbal behaviour: function and neurological considerations. Aphasiology 4, 133-154. doi: 10.1080/02687039008249066

Xing, S., Lacey, E. H., Skipper-Kallal, L. M., Jiang, X., Harris-Love, M. L., Zeng, J., et al. (2016). Right hemisphere grey matter structure and language outcomes in chronic left hemisphere stroke. Brain 139, 227-241. doi: 10.1093/brain/awv323

Conflict of Interest Statement: The authors declare that the research was conducted in the absence of any commercial or financial relationships that could be construed as a potential conflict of interest.

Copyright (C) 2017 Berthier, Torres-Prioris and López-Barroso. This is an open-access article distributed under the terms of the Creative Commons Attribution License (CC $B Y)$. The use, distribution or reproduction in other forums is permitted, provided the original author(s) or licensor are credited and that the original publication in this journal is cited, in accordance with accepted academic practice. No use, distribution or reproduction is permitted which does not comply with these terms. 